\title{
Simbolismo e naturalismo fra lingua e testo, a cura di Sergio Cigada e Marisa Verna
}

\section{Maria Emanuela Raffi}

\section{(2) OpenEdition}

1 Journals

\section{Edizione digitale}

URL: http://journals.openedition.org/studifrancesi/6056

DOI: 10.4000/studifrancesi.6056

ISSN: 2421-5856

\section{Editore}

Rosenberg \& Sellier

\section{Edizione cartacea}

Data di pubblicazione: 1 mai 2011

Paginazione: 201-203

ISSN: 0039-2944

\section{Notizia bibliografica digitale}

Maria Emanuela Raffi, «Simbolismo e naturalismo fra lingua e testo, a cura di Sergio Cigada e Marisa Verna», Studi Francesi [Online], 163 (LV | I) | 2011, online dal 30 novembre 2015, consultato il 09 janvier 2021. URL: http://journals.openedition.org/studifrancesi/6056 ; DOI: https://doi.org/10.4000/ studifrancesi.6056

Questo documento è stato generato automaticamente il 9 janvier 2021.

\section{(c)}

Studi Francesi è distribuita con Licenza Creative Commons Attribuzione - Non commerciale - Non opere derivate 4.0 Internazionale. 


\title{
Simbolismo e naturalismo fra lingua e testo, a cura di Sergio Cigada e Marisa Verna
}

\author{
Maria Emanuela Raffi
}

\section{NOTIZIA}

AA. VV., Simbolismo e naturalismo fra lingua e testo, a cura di Sergio CIGADA e Marisa VERNA, Milano, Vita e Pensiero, 2010, pp. 522.

1 Questo ricco volume di contributi, prodotto dell'omonimo Convegno che ha avuto luogo a Milano nel 2003, ha assunto un valore ed un significato particolari con la scomparsa di sergio CIGADA, ispiratore della serie di Convegni sul simbolismo tenuti a Milano e a Pescara dal 1992 al 2003 e autore della prefazione. Dedicato a Cigada dalla curatrice Marisa Verna, il volume ne raccoglie in buona parte l'ampia eredità di riflessioni critiche, ribadite anche nel corposo contributo che rappresenta, ora a maggior ragione, il cuore della pubblicazione: Rimbaud dalla "Lettre du Voyant" a "Le Bateau ivre" (pp. 87-128). L'articolo costituisce l'occasione, per Cigada, di dare un'articolata sistemazione ai concetti fondamentali della lettera del 15 maggio: storia della poesia dall'antica Grecia, il «dérèglement», il «Voyant», l'«Inconnu», «trouver une langue». Sotto il segno di un Rimbaud molto 'baudelairiano', che con la voyance "giunge alle radici dello spirito», Cigada traccia un percorso in cui l'esperienza della Comune appare come una «deriva politica» transitoria e chiusa e in cui «Le Bateau ivre» sembra riprendere perfettamente la struttura e le preoccupazioni di rinascita della poesia della «Lettre du Voyant»: ascesa fino all'Inconnu e ridiscesa in forme poetiche rinnovate. «Completamente legato allo schema parnassiano» per quanto riguarda la forma metrica, «Le Bateau ivre» realizzerebbe un concetto simbolista fondamentale per Cigada: «l'idea dell'atto poetico come l'atto supremo, atto trascendente, strumento di conoscenza dell'Infinito, dell'Assoluto, dell'Ignoto». 
2 Ripartiti fra simbolismo e naturalismo, gli altri 23 contributi sono organizzati in cinque parti.

La prima, «Fra Simbolismo e Naturalismo», è inaugurata da Giovanna BELLATI (Le renouveau du rondeau entre Parnasse et Symbolisme, pp. 3-30) che analizza le modalità della ripresa del rondeau nell'opera di alcuni importanti poeti da Banville a Mendès.

Sempre alla 'forma poetica' è dedicato lo studio di olivier BIVORT, Les conditions linguistiques de la modernité simboliste (pp.31-49), che individua nel verso «l'objectif principal de la bataille symboliste». Ciò che è messo in causa dai simbolisti è per Bivort il codice stesso dell'espressione verbale, il legame riconosciuto fra idea e segno, in nome di una più libera e moderna espressività nella lingua, ma soprattutto nella poesia. Giuseppe BERNARDELLI propone in Baudelaire naturalista (pp. 51-85) una dettagliata analisi del testo liminare «Au lecteur» per mostrare come tutto il volgare apparato analogico che lo costituisce appartenga alla «categoria della degradazione al fisiologico e al biologico dei fatti spirituali». Tutto il successivo percorso di Bernardelli attraverso le Fleurs tende a interpretare la forte presenza di questi "elementi naturalistici" come espressione dello spleen e della concezione baudelairiana dell'uomo visto come «spirito che si agita dentro la materia in cui si è misteriosamente trovato imprigionato».

Dopo il lungo saggio di sergio CIGADA di cui si è già riferito, Françoise GALLARD (Entre mythe et savoir: le naturalisme de Zola, pp. 129-137) analizza la coesistenza, nell'opera di Zola, di un linguaggio simbolico - veicolo del mito e in particolare del fondamentale mito delle origini - e del linguaggio naturalistico, che tende a storicizzare il mito nel segno dell'ereditarietà.

7 Nel contributo successivo, Paola PAISSA presenta uno studio dell'« écriture impressioniste " in La Curée di Zola. Alle caratteristiche di questo tipo di scrittura, illustrate nella prima parte, segue l'analisi della specifica « écriture androgyne » del romanzo zoliano (Symbolisme et naturalisme dans "La Curée" de Zola, pp. 139-158).

8 Mario PETRONE conclude la prima parte con un'incursione nelle letture di un personaggio di La Faute de l'abbé Mouret, Jeanbernat (La bibliothèque imaginaire des "Rougon-Macquart". L'exemple de Jeanbernat lecteur de d'Holbach, pp. 159-168).

9 La seconda parte, dedicata al «Fantastico», si apre con un saggio di Liana NISSIM sulle Forme del meraviglioso cristiano nella lirica simbolista (pp.171-196), in cui viene tracciato il percorso della «scrittura simbolista del meraviglioso cristiano» da Baudelaire e Flaubert a Mallarmé, Lorrain, Samain e infine a Huysmans, utilizzando come chiave di lettura l'attrazione comune per «la pratica dell'ekphrasis», che da À une Madone a Hérodiade a Là-bas, attraverso le variazioni e le stilizzazioni di diversi autori simbolisti, riproduce con toni dal drammatico al quasi auto-parodico alcuni dei temi maggiori dell'iconografia cristiana.

10 In Scienza e visione, tecnologia e artificiale. Automi, androidi e robots fra la fine dell'ottocento e il primo Novecento (pp.197-216), Marco MODENESI affronta il tema della 'modernità' e dell'artificiale, di cui traccia le origini letterarie ed extraletterarie, per soffermarsi poi particolarmente sui romanzi di Leroux e soprattutto sull'Ève future di Villiers de l'IsleAdam, nella quale riconosce una chiara matrice baudelairiana.

11 La diffusa presenza e le particolari caratteristiche della ripresa della figura di Narciso alla fine dell'Ottocento, «noyau archétypal du décadentisme», sono attentamente indagate nell'ampia ricognizione critica di Ida MERELlo (De Narcisse à la sirène. Variations sur des thèmes mythiques dans l'imaginaire fin de siècle, pp. 217-232). L'A., individuati gli 
elementi profondi e costitutivi del mito - «le reflet» e «la perception de l'altérité de l'image» - li confronta con la singolare "valence renversée d'anti-Narcisse» e con il conseguente e angosciante sdoppiamento della personalità che il mito stesso assume in alcune opere significative di fine secolo: Le Roi au masque d'or di Schwob, Le Horla di Maupassant, Claire Lenoir di Villiers de l'Isle-Adam, Chemin nuptial di Scheffer.

Il fantastico fin de siècle come "véhicule privilégié des angoisses et des terreurs qui affligent l'homme» è al centro dello studio di simonetta VALENTI, Un saut dans le mystère. Aspects structurels du conte mythologique décadent (Lorrain, Mauclair, Schwob, pp. 233-260), che rileva, attraverso l'analisi di Le Roi au masque d'or, Narkiss e Le Bouclier d'Or, importanti analogie che portano all'ipotesi di un genere unitario, il «récit mythologique décadent», collocato in un tempo immemoriale $\mathrm{e}$ in un luogo impenetrabile.

13 In Jean Lorrain e il canovaccio fantastico (pp.261-279), Marilia MARCHETTI analizza procedimenti con i quali l'autore introduce l'ironia nei suoi racconti fantastici: sottolineature tipografiche come il corsivo, il colore delle lettere o retoriche quali l'enumerazione, la ripetizione o ancora l'iperbole e l'uso di «stereotipi tematici» che arrivano fino alla maschera vera e propria.

14 Ruggero CAMPAGNOLI chiude la seconda parte con Fantastico e preconscio (pp. 281-295), in cui approfondisce la tripartizione (merveilleux, étrange, fantastique) proposta da Todorov, mettendola in contatto con le tripartizioni indicate da Vico (sentire, avvertire e riflettere) e soprattutto da Freud (conscio, preconscio e inconscio) e affermando quindi l'esistenza di un'affinità fra preconscio e fantastico, che viene verificata nella lettura del Cycle patibulaire di Georges Eekhoud.

Il contributo di Mariella DI MAIO (Les «petites tragédies» de Barbey. Sur "Les Diaboliques", pp. 299-309) apre la terza parte, «Teatro». Les Diaboliques di Barbey vi sono analizzate come pièces di un «théâtre des passions: des passions secrètes, cachées, sans nom», di cui sono puntualmente indagati gli inquietanti segni infernali.

Bristina BRANCAGLION (Il francese tecnico-scientifico nel dramma realista e naturalista, pp. 311-334) sposta l'attenzione sulla lingua teatrale del secondo Ottocento per indagare un fenomeno specifico, quello del teatro finanziario, che da Marcadet di Balzac a Les Ventres dorés di Emile Fabre presenta un lessico talmente connotato da riuscire a definire, con la sua sola presenza, l'ambiente socio-professionale cui il personaggio appartiene.

17 In Spazi, aspirazioni e trasgressioni del teatro naturalista in scena (pp. 335-342), Mariangela MAZZOCCHI DOGLIO mette in luce le caratteristiche innovative del teatro naturalista: allargamento dello spazio al «fuori scena», scelta della forma breve, rinnovamento tematico e, soprattutto, valorizzazione della funzione del regista, resa fondamentale da André Antoine.

Al teatro simbolista e alle sue convergenze con la pittura di fine secolo, soprattutto preraffaellita, è dedicato invece lo studio di Rosalba GASPARRO, Fughe e variazioni. Forme della parola simbolica nel teatro di Maeterlinck (pp. 343-364), che prende avvio dall'analisi de La Princesse Maleine e dal passaggio del personaggio vivente all'oggetto inanimato che la «figurina di cera» della protagonista porta sulla scena. Le opere successive, fino a Pelléas et Mélisande e oltre fino a L'Oiseau bleu, confermano per l'A. una "poetica dell'assenza e del riflesso» propria della marionetta, insieme alla costante ispirazione fiabesca e pittorica. 

et picturales, pp. 405-424). espressionista. vision subjective/temporelle».

Eleonora SPARVOLI propone, in chiusura della terza parte, una ricognizione sulla presenza del Lohengrin di Wagner nell'opera di molti autori della seconda metà dell'Ottocento, da Baudelaire a Nietzsche, agli autori della «Revue wagnerienne» - fra cui Mallarmé - e infine a Laforgue, il cui Lohengrin, lacerato nella sua compattezza analogica e punteggiato di giochi verbali e di citazioni, finisce col distruggere il suo stesso modello (Il "Lohengrin" di Richard Wagner: tema con variazioni, pp. 365-378).

Nella quarta parte, «Arte», la pittura appare subito in primo piano in Parler peinture di carminella SIPALA (pp.381-404), dedicato alla critica d'arte nella seconda metà dell'Ottocento e particolarmente al «discours métapictural» di Huysmans e di Zola, dei quali analizza le modalità di osservazione e soprattutto di descrizione, la transcription d'art, con le sue evidenti ricadute sul pubblico dell'epoca.

Dedicato all' «observation de quelques œuvres littéraires et picturales déclinant le thème urbain», lo studio di Maria Teresa MOIA prende in esame la novella Antoine Mathieu di Paul Heusy, che collega alla tela Le moulin à café di Charles De Groux, poi La Ville di Rodenbach avvicinata a La nuit à Bruges di Degouve de Nuncques, tracciando così il contemporaneo passaggio di letteratura e pittura in Belgio dal naturalismo al simbolismo (L'image de la ville belge entre naturalisme et symbolisme. Perspectives narratives

2 Il difficile rapporto fra Émile Zola e la pittura di Gustave Moreau è l'oggetto del saggio di Annamaria LASERRA, che riconosce significative somiglianze fra la Salomé di Moreau, che nel 1876 aveva sconcertato e irritato lo scrittore, e il romanzo zoliano L'Euvre (1886) in cui Claude Lantier mette sulla tela una figura femminile immaginaria e sognata (Zola et la «Salomé» de Gustave Moreau: le défi du Sphinx, pp. 425-435).

Con Naturalismo di Zola tra impressionismo ed espressionismo (pp.437-455), Maria Immacolata SPAGNA chiude la parte dedicata all'«Arte» tracciando «le più importanti tappe dell'evoluzione della poetica naturalista di Zola» attraverso i contatti con gli impressionisti prima, la cui originale interpretazione della realtà lo affascinava, con gli espressionisti poi. In assenza della preziosa critica d'arte zoliana fra il 1881 e il 1895, è nei romanzi apparsi dopo il 1880 che l'A. ritrova le cupe suggestioni dell'estetica

4 Dedicata alla «Fortuna», la quinta e ultima parte della raccolta propone anzitutto il contributo di Mariacristina PEDRAZZINI, L'écriture naturiste (pp. 459-475) che sonda le forme poetiche e le scelte tematiche e formali di un movimento letterario nato proprio alla fine del secolo, il «naturisme», prendendo in esame soprattutto gli scritti di SaintGeorges de Bouhélier e di Maurice Le Blond.

In D'une chevelure odorante au temps de l'extase. Sur des synesthésies proustiennes (pp. 477-494), Marisa VERNA si colloca fra il xIX e il xx secolo per definire la particolare reinterpretazione che Proust propone nella sua opera delle «analogies inspiratrices» di Baudelaire. In questo passaggio, tuttavia, la figura della «synesthésie» subisce una trasformazione fondamentale, passando «de la dimension objective/métaphysique à la

Francesco DI PILLA chiude il volume con uno studio su Richiami positivisti e suggestioni simboliche tra Francia e Italia nella narrativa di Grazia Deledda (pp. 495-522) nel quale cerca di cancellare i due luoghi comuni più diffusi-l'«incultura» della scrittrice e il peso della situazione della Sardegna dell'epoca-sottolineando significative influenze 
francesi come quelle di Bourget e Zola, che, assieme alla personale sensibilità per il mistero della Deledda, convergono a definirne la dimensione europea. 